\title{
Vitrectomy for advanced proliferative diabetic retinopathy: 30 years on
}

Tock H. Lim ${ }^{1}$, Louis Lim¹, Mae-Lynn Bastion²

${ }^{1}$ National Healthcare Group Eye Institute, Tan Tock Seng Hospital, Singapore; ${ }^{2}$ Department of Ophthalmology, Faculty of Medicine, Universiti Kebangsaan Medical Centre, Kuala Lumpur, Malaysia

The scourge of diabetes mellitus continues to ravage the world. South East Asia ranks second among seven geographical regions in absolute numbers of patients with diabetes. ${ }^{1}$ In Malaysia, the prevalence of type 2 diabetes mellitus (T2DM) has risen progressively from $11.6 \%$ in 2006 , to $15.2 \%$ in $2011,{ }^{2}$ and $17.5 \%$ in $2015 .{ }^{3}$ Globally, diabetic retinopathy continues to be among the top five causes of moderate to severe visual impairment. ${ }^{4}$ It is among the top three in Malaysia. ${ }^{5}$ Globally, about a quarter of T2DM suffers from diabetic retinopathy. ${ }^{6}$ Among diabetic patients registered in the Diabetic Eye Registry of Malaysia in 2007, 36\% suffers from diabetic retinopathy, $15 \%$ from sight-threatening retinopathy, and $7 \%$ from proliferative diabetic retinopathy (PDR). ${ }^{?}$

The Diabetic Retinopathy Vitrectomy Study (DRVS) was the first landmark study comparing vitrectomy vs observation among patients with advanced PDR. Published in 1985, DRVS Report 2 found no difference in outcome between early vitrectomy vs deferring for a year for vitreous haemorrhage secondary to PDR of T2DM with baseline vision of 5/200 or worse. ${ }^{8}$ Early vitrectomy was advantageous for PDR patients with useful vision but mainly those with extensive neovascularization. ${ }^{9}$ In fact, $20 \%$ in both arms of the study had lost light perception by 2 years.

Over the last three decades, significant advancements have contributed to better outcomes for patients with PDR: better laser photocoagulators, anti-vascular endothelial growth factors (anti-VEGF), and better vitrectomy instrumentation. ${ }^{10}$ Used in combination, vitrectomy surgeons have achieved progressively better outcomes for advanced PDR patients, ${ }^{11}$ with anatomic success rates improving from $66-88 \%$ (prior to 1990 ) to $83.0-92.6 \%$ (after 2000) and less than $25 \%$ of patients suffering vision loss. ${ }^{2}$

Surgical approaches to diabetic tractional retinal detachment (DM-TRD) have advanced over the years from membrane peeling and segmentation, to delamination (with either en bloc dissection ${ }^{13}$ or bimanual technique ${ }^{14}$ ), and the shaving technique. ${ }^{15}$ For a young vitrectomy surgeon just starting out, these techniques may 
seem confusing - which should I learn and which shall I use? As DM-TRD comes in various configurations, these techniques are best understood as different tools in the surgeon's toolbox. In the following paragraphs, I will summarise them and propose a unified approach based on the pathogenesis of DM-TRD.

The vasoproliferative action of VEGF causes new endothelial buds to sprout from existing vessels, crossing the internal limiting membrane to adhere to the posterior hyaloid, forming vascular pegs and holding the posterior hyaloid down onto the retinal surface. These vascular pegs are concentrated along the larger retinal vessels, especially the vascular arcades and first two orders of retinal vessels radiating from the optic disc and the arcades. As the posterior hyaloid separates, these vascular pegs prevent posterior vitreous detachment (PVD) from occurring normally. Forces of partial PVD act on these vascular pegs, causing them to bleed. As a result, scar tissue bridges these pegs to form tractional membranes. Tractional membranes along the vascular arcades give rise to the typical wolf-jaw configuration. The tractional membranes and posterior hyaloid that span across the superior and inferior arcades form the table-top traction. Tractional membranes running along the radial vessels form radial tractional membranes. As the radial vessels taper towards the retinal periphery, the vascular pegs become tinier and weaker in the periphery, allowing more hyaloid separation to occur beyond the retinal mid-periphery. Posterior hyaloid traction bridging the wolf-jaw traction and the vitreous base form anteroposterior (AP) traction. ${ }^{16}$

Hence, a unified concept of vitrectomy for diabetic TRD is to remove the anchoring forces of the vascular pegs, thereby allowing complete PVD to take place from the posterior pole to the periphery, using whatever techniques available at the surgeon's disposal. ${ }^{17}$

However, when confronted with a myriad of different DM-TRD configurations, the young vitrectomy surgeon often asks: where do I start? Between the temporal termination of the superior and inferior vascular arcades, the surgeon can usually find a surgical plane between the diabetic tractional membrane and the retina, other than cases of table-top TRD (Fig. 1a). This is due to the lack of larger retinal blood vessels at the temporal macular edge and macula, hence the paucity of vascular pegs. Through the temporal entry point, one can remove the posterior hyaloid over the macula with ease. Next, the surgeon could work along the vascular arcades (Fig. 1b). If the pegs are far apart, segmentation is quick and effective. If the pegs are close together, the surgeon can employ delamination techniques. With the en bloc method, the AP traction is left untouched, using it as the "third hand", while with the bimanual method, the membrane is lifted with a pair of forceps in one hand, cutting the pegs with scissors with the other, aided by chandelier lighting. ${ }^{18}$

The shaving technique is particularly useful for broad sheets of membrane with multiple, tightly spaced vascular pegs where the delamination technique would be very time consuming. The shaving technique is made possible by small gauge vitrectors (25-G or $27-\mathrm{G}$ ) where the ports are located very close to the tip. ${ }^{11}$ 

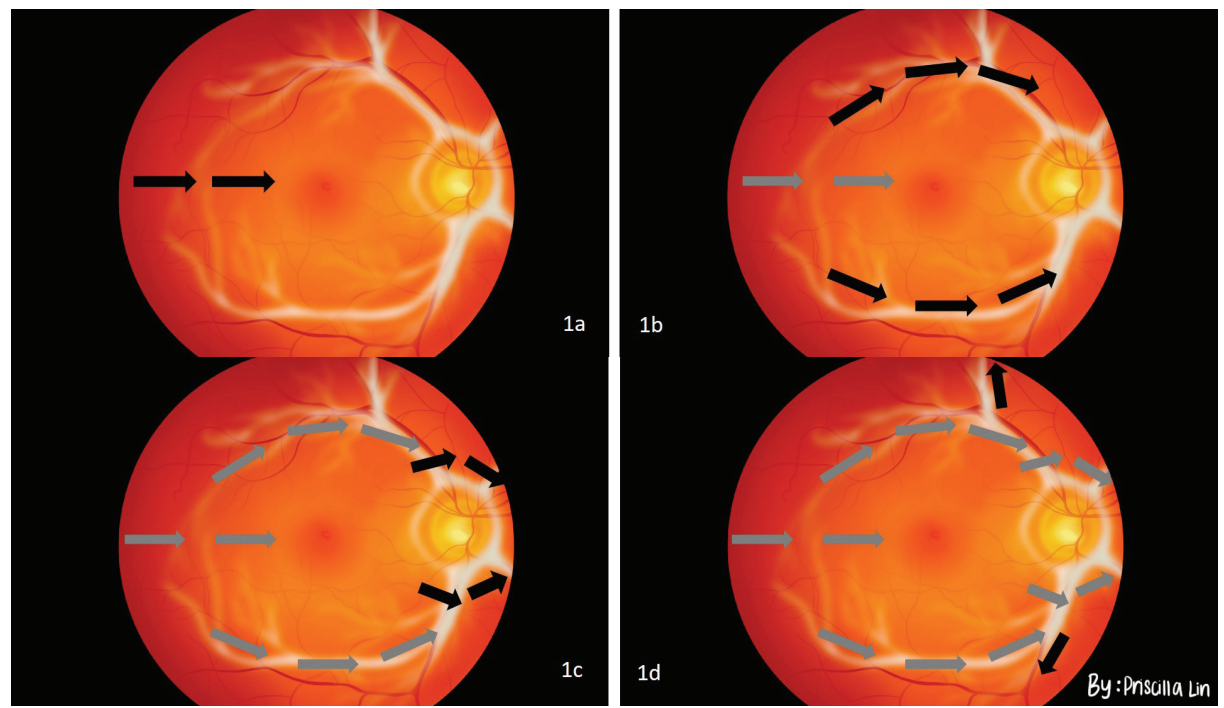

Fig. 1. Sequential surgical approach for diabetic tractional membranes: (a) temporal macula entry point; (b) dissection along the superior and inferior arcades; (c) dissection around the optic nerve head; and $(d)$ dissection along the radial traction bands.

In addition, the smaller port opening offers better flow control (port-based flow limitation). Combined with high-speed cutting, ${ }^{19}$ the surgeon can exercise flow control with exquisite accuracy using the full stroke length of the pedal. On the other hand, it is advisable to skirt around the optic nerve head, as the membranes there tend to bleed profusely (Fig. 1c).

Once the posterior hyaloid is mobilised around the vascular arcade, dissection takes place along the radial traction band (Fig. 1d). Once released, the posterior hyaloid is engaged using the vitreous cutter, extending the PVD centripetally. In the mid-periphery, the vascular pegs are often sparse and weak. This is where membrane peeling is most useful.

When TRD is combined with rhegmatogenous retinal detachment and/or proliferative vitreoretinopathy, meticulous peripheral vitreous shaving with indentation is necessary. In these cases, 23-G cutters may provide better counterforce for indentation.

Preoperative anti-VEGF injection is one of the most important innovations for diabetic vitrectomy. It has been shown to reduce intraoperative haemorrhage, iatrogenic breaks, ${ }^{20}$ post-vitrectomy haemorrhage,${ }^{21}$ and operation time.${ }^{22}$ Although anti-VEGF injection is known to worsen TRD, it is uncommon. Occurring in approximately $5 \%$ of cases ${ }^{23}$ and not higher than controls, it should not deter the use of anti-VEGF injections preoperatively. ${ }^{24}$

In this issue of Malaysian Journal of Ophthalmology, Dr. Mushawiahti Mustapha 
showed that excellent surgical results can be achieved using small-gauge vitrectomy, modern viewing and lighting systems, and preoperative intravitreal bevacizumab injections. ${ }^{25}$ In this retrospective case series, more than $96 \%$ of cases with diabetic TRD achieved anatomical success, and $85 \%$ achieved visual stabilization or improvement at 1 year. These excellent surgical results would serve to encourage early presentation and early referral for surgical intervention. ${ }^{26}$

Just as surgical outcomes are improving, intravitreal pharmacotherapy is also conquering new grounds for PDR management. ${ }^{27}$ Intravitreal anti-VEGF therapy for PDR, including those with vitreous haemorrhage ${ }^{28}$ and TRD not involving the macula, can achieve surprisingly good results. In a short randomized controlled trial, three consecutive monthly doses of ranibizumab injections reduced the 4-month cumulative vitrectomy rate from $17 \%$ to $12 \% .{ }^{29}$ Persistence in delivering anti-VEGF therapy and judicious use of panretinal photocoagulation can help some patients achieve long-term stability of the retinopathy without surgery. ${ }^{30}$ For the vitrectomy surgeon, this poses another dilemma: in cases of vitreous haemorrhage and DM-TRD without obvious macular involvement, should we offer early surgical intervention or intravitreal anti-VEGF therapy? ${ }^{31}$

Thirty years on, it is perhaps time for another major diabetic retinopathy vitrectomy study. We eagerly await the results of DRCR.net Protocol AB. ${ }^{32}$

\section{References}

1. Saeedi P, Petersohn I, Salpea P, et al. Global and regional diabetes prevalence estimates for 2019 and projections for 2030 and 2045: Results from the International Diabetes Federation Diabetes Atlas, 9th edition. Diabetes Res Clin Pract. 2019;157:107843.

2. Jan Mohamed HJ, Yap RW, Loy SL, Norris SA, Biesma R, Aagaard-Hansen J. Prevalence and determinants of overweight, obesity, and type 2 diabetes mellitus in adults in Malaysia. Asia Pac J Public Health. 2015;27(2):123-135.

3. Tee ES, Yap RWK. Type 2 diabetes mellitus in Malaysia: current trends and risk factors. Eur J Clin Nutr. 2017;71(7):844-849.

4. Flaxman SR, Bourne RRA, ResnikoffS, et al. Global causes of blindness and distance vision impairment 1990-2020: a systematic review and meta-analysis. Lancet Glob Health. 2017;5(12):e1221-e1234.

5. Chew FLM, Salowi MA, Mustari Z, et al. Estimates of visual impairment and its causes from the National Eye Survey in Malaysia (NESII). PLoS One. 2018;13(6):e0198799. Published 2018 Jun 26.

6. Thomas RL, Halim S, Gurudas S, Sivaprasad S, Owens DR. IDF Diabetes Atlas: A review of studies utilising retinal photography on the global prevalence of diabetes related retinopathy between 2015 and 2018. Diabetes Res Clin Pract. 2019;157:107840.

7. Goh PP, National Eye Database Study Group. Status of diabetic retinopathy among diabetics registered to the Diabetic Eye Registry, National Eye Database, 2007. Med J Malaysia. 2008;63(Suppl C):24-28. 
8. Early vitrectomy for severe vitreous hemorrhage in diabetic retinopathy. Two-year results of a randomized trial. Diabetic Retinopathy Vitrectomy Study report 2. The Diabetic Retinopathy Vitrectomy Study Research Group. Arch Ophthalmol. 1985;103(11):1644-1652.

9. Early vitrectomy for severe proliferative diabetic retinopathy in eyes with useful vision. Results of a randomized trial. Diabetic Retinopathy Vitrectomy Study Report 3. The Diabetic Retinopathy Vitrectomy Study Research Group. Ophthalmology. 1988;95(10):1307-1320.

10. Mansour SE, Browning DJ, Wong K, Flynn HW Jr, Bhavsar AR. The Evolving Treatment of Diabetic Retinopathy. Clin Ophthalmol. 2020;14:653-678.

11. Sokol JT, Schechet SA, Rosen DT, Ferenchak K, Dawood S, Skondra D. Outcomes of vitrectomy for diabetic tractional retinal detachment in Chicago's county health system. PLoS One. 2019;14(8):e0220726.

12. Stewart MW, Browning DJ, Landers MB. Current management of diabetic tractional retinal detachments. Indian J Ophthalmol. 2018;66(12):1751-1762.

13. Kakehashi A. Total en bloc excision: a modified vitrectomy technique for proliferative diabetic retinopathy. Am J Ophthalmol. 2002;134(5):763-765.

14. Iyer SSR, Regan KA, Burnham JM, Chen CJ. Surgical management of diabetic tractional retinal detachments. Surv Ophthalmol. 2019;64(6):780-809.

15. Berrocal MH. All-Probe Vitrectomy Dissection Techniques for Diabetic Tractional Retinal Detachments: Lift and Shave. Retina. 2018;38(Suppl 1):S2-S4.

16. Hutton WL, Bernstein I, Fuller D. Diabetic traction retinal detachment. Factors influencing final visual acuity. Ophthalmology. 1980;87(11):1071-1077.

17. Gündüz K, Bakri SJ. Management of proliferative diabetic retinopathy. Compr Ophthalmol Update. 2007;8(5):245-256.

18. Elwan MM, Hagras SM, Ellayeh AA. Trimanual Versus Unimanual 23-Gauge Vitrectomy in Patients With Diabetes: Limitations and Expectations. Ophthalmic Surg Lasers Imaging Retina. 2019;50(1):42-49.

19. RizzoS,Genovesi-EbertF,BeltingC.Comparativestudybetweenastandard25-gaugevitrectomysystem and a new ultrahigh-speed 25-gauge system with duty cycle control in the treatment of various vitreoretinal diseases. Retina. 2011;31(10):2007-2013.

20. Arevalo JF, Lasave AF, Kozak I, et al. Preoperative Bevacizumab for Tractional Retinal Detachment in Proliferative Diabetic Retinopathy: A Prospective Randomized Clinical Trial. Am J Ophthalmol. 2019;207:279-287.

21. Ahmadieh H, Shoeibi N, Entezari M, Monshizadeh R. Intravitreal bevacizumab for prevention of early postvitrectomyhemorrhage in diabetic patients: a randomized clinical trial. Ophthalmology. 2009;116(10):1943-1948.

22. Zhao LQ, Zhu H, Zhao PQ, Hu YQ. A systematic review and meta-analysis of clinical outcomes of vitrectomy with or without intravitreal bevacizumab pretreatment for severe diabetic retinopathy. $\mathrm{Br} J$ Ophthalmol. 2011;95(9):1216-1222.

23. Arevalo JF, Maia M, Flynn HW Jr, et al. Tractional retinal detachment following intravitreal bevacizumab (Avastin) in patients with severe proliferative diabetic retinopathy. $\mathrm{Br} \mathrm{J}$ Ophthalmol. 2008;92(2):213-216. 
24. Bressler NM, Beaulieu WT, Bressler SB, et al. Anti-Vascular Endothelial Growth Factor Therapy and Risk of Traction Retinal Detachment in eyes with Proliferative Diabetic Retinopathy: Pooled Analysis of Five DRCR Retina Network Randomized Clinical Trials. Retina. 2020;40(6):1021-1028.

25. Mushawiahti M, Tan CY, Tevanthiran G, et al. Small-gauge vitrectomy for advanced diabetic eye disease: Outcome and predictive factors for post-operative poor vision. Malaysian Journal of Ophthalmology. 2020; 2(3):177-189.

26. Stewart MW, Browning DJ, Landers MB. Current management of diabetic tractional retinal detachments. Indian J Ophthalmol. 2018;66(12):1751-1762.

27. Gross JG, Glassman AR, Liu D, et al. Five-Year Outcomes of Panretinal Photocoagulation vs Intravitreous Ranibizumab for Proliferative Diabetic Retinopathy: A Randomized Clinical Trial [published correction appears in JAMA Ophthalmol. 2019 Apr 1;137(4):467]. JAMA Ophthalmol. 2018;136(10):11381148.

28. Wirkkala J, Bloigu R, Hautala NM. Intravitreal bevacizumab improves the clearance of vitreous haemorrhage and visual outcomes in patients with proliferative diabetic retinopathy. BMJ Open Ophthalmol. 2019;4(1):e000390.

29. Diabetic Retinopathy Clinical Research Network ${ }^{\star}$. Randomized clinical trial evaluating intravitreal ranibizumab or saline for vitreous hemorrhage from proliferative diabetic retinopathy. JAMA Ophthalmol. 2013;131(3):283-293.

30. Yang CS, Hung KC, Huang YM, Hsu WM. Intravitreal bevacizumab (Avastin) and panretinal photocoagulation in the treatment of high-risk proliferative diabetic retinopathy. J Ocul Pharmacol Ther. 2013;29(6):550-555.

31. Lin J, Chang JS, Yannuzzi NA, Smiddy WE. Cost Evaluation of Early Vitrectomy versus Panretinal Photocoagulation and Intravitreal Ranibizumab for Proliferative Diabetic Retinopathy. Ophthalmology. 2018;125(9):1393-1400.

32. ClinicalTrials.gov. Anti-VEGF vs. Prompt Vitrectomy for VH From PDR (DRCR.net Protocol AB). 